\section{Acute hyperglycemia induces an oxidative stress in healthy subjects}

Recent prospective studies indicate that long-term glycemic control of diabetes is an important predictor not only of microvascular disease but also of macrovascular complications, including coronary heart disease (1). Possible links between glucose and cardiovascular events in the diabetic patient include modifications of important vascular functions of the endothelium with a switch from a quiescent, relaxant, antithrombotic, antioxidant, and antiadhesive state to an activated state displaying a more atherogenetic risk profile (2). Generation of reactive oxygen species could be a common downstream mechanism by
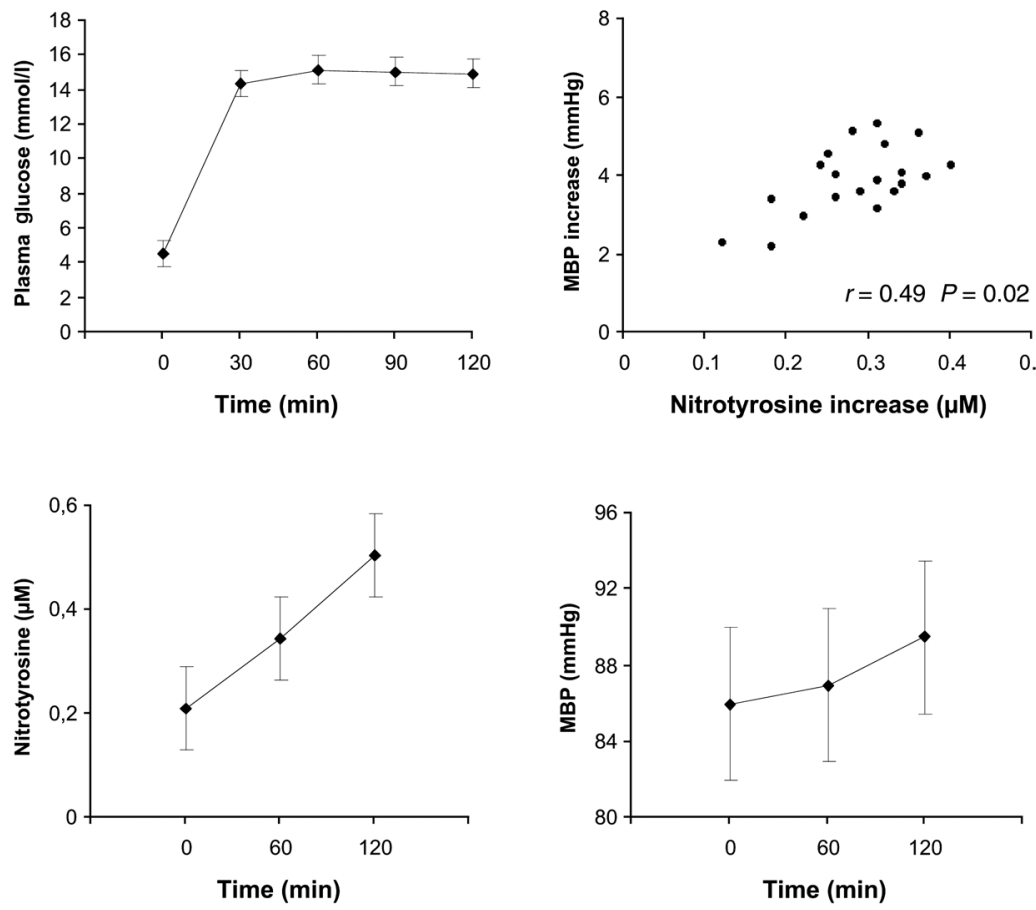

Figure 1

Twenty healthy subjects were submitted to a hyperglycemic glucose clamp study in which plasma glucose levels were acutely raised to $15 \mathrm{mmol} / \mathrm{l}(0.33 \mathrm{~g} / \mathrm{kg}$ as intravenous bolus injection followed by a variable $30 \%$ glucose infusion). Mean blood pressure (MBP) was calculated as diastolic plus one-third pulse pressure. Nitrotyrosine was assayed according to Ter Steege et al. (5): the standard curve was constructed with serial dilution of a nitrated protein solution; glucose interference was excluded by performing the ELISA assay of standard solution in the presence of various glucose concentrations (from 10 to $100 \mathrm{mmol} / \mathrm{l}$ ); the limit of detection of the assay was $10 \mathrm{nmol} / \mathrm{l}$, with intra- and interassay coefficient of variations of $4.5 \%$ and $8 \%$, respectively. Nitrotyrosine levels rose steadily during hyperglycemia and remained significantly above baseline at the end of the study. The correlation between nitrotyrosine and MBP increases during the clamp suggests that the two phenomena are related and strictly dependent upon ongoing hyperglycemia. Data are mean \pm SD . cose clamp test in which plasma glucose concentrations were acutely raised at about $15 \mathrm{mmol} / \mathrm{l}$ for 120 minutes (Figure 1). Mean blood pressure and nitrotyrosine (5) rose significantly during the clamp; there was a positive correlation $(r=0.49)$ between nitrotyrosine and mean blood pressure increases during hyperglycemia. In control studies $(n=6)$, in which plasma glucose was maintained at normal concentrations ( $5 \mathrm{mmol} / \mathrm{l}$ for 120 minutes), we could detect no variation in nitrotyrosine plasma levels from baseline (baseline: $0.15 \pm 0.05 \mu \mathrm{mol} / 1 ; 120$-minute values: $0.13 \pm 0.05 \mu \mathrm{mol} / 1, P=$ not significant $)$. We show here that acute hyperglycemia in normal subjects causes an oxidative stress as evidenced by the raised circulating nitrotyrosine levels during the hyperglycemic clamp. However, we cannot exclude the possibility that some nitrotyrosine can be generated via a peroxynitrite-independent mechanism, or that a reduced nitrotyrosine clearance during hyperglycemia could also contribute to its raised plasma concentrations. Since nitrotyrosine is considered a good marker of peroxynitrite formation (6), and since peroxynitrite may account for a considerable portion of the toxic effects previously attributed to nitric oxide or the superoxide anion (7), it is possible that some of the toxic effects of hyperglycemia on the vascular tree may be modulated by peroxynitrite. Acute hyperglycemia in normal subjects may in fact induce vasoconstriction, activate thrombosis, increase the circulating levels of soluble adhesion molecules, and prolong the QT interval $(8,9)$. The recent demonstration (10) that apoptosis of myocytes, endothelial cells, and fibroblasts in heart biopsies taken from diabetic patients is selectively associated with intracellular levels of nitrotyrosine supports a role for high-energy oxidants (such as peroxynitrite) as mediators of the vascular damage brought about by hyperglycemia.

Raffaele Marfella, ${ }^{1}$

Lisa Quagliaro, ${ }^{2}$

Francesco Nappo, ${ }^{1}$

Antonio Ceriello, ${ }^{2}$

and Dario Giugliano 
${ }^{1}$ Department of Geriatrics and Metabolic Diseases, Second University of Naples, Naples, Italy

${ }^{2}$ Department of Pathology and Medicine, Experimental and Clinical, Division of Internal Medicine, University of Udine, Udine, Italy

Address correspondence to: Dario Giugliano, Department of Geriatrics and Metabolic Diseases, Piazza L. Miraglia, 80031 Naples, Italy. Phone/Fax: 39-081-5665054; E-mail: dario.giugliano@unina2.it.

1. Capes, S.E., Hunt, D., Malmberg, K., and Gerstein, H.C. 2000. Stress hyperglycaemia and increased risk of death after myocardial infarction in patients with and without diabetes: a systematic overview. Lancet. 355:773-778.

2. Giugliano, D., et al. 1997. Vascular effects of acute hyperglycemia in humans are reversed by L-arginine. Evidence for reduced availability of nitric oxide during hyperglycemia. Circulation. 95: $1783-1790$

3. Nishikawa, T., et al. 2000. Normalizing mithocondrial superoxide production blocks three pathways of hyperglycaemic damage. Nature. 404:787-790.

4. Pennathur, S., Wagner, J.D., Leeuwenburgh, C., Litwak, K.N., and Heinecke, J.W. 2001. A hydroxyl radical-like species oxidizes cynomolgus monkey artery wall proteins in early diabetic vascular disease. J. Clin. Invest. 107:853-860.

5. Ter Steege, J.C.A., Koster-Kamphuis, L., Vas Straaten, E., Forget, P., and Buurman, W.A. 1998 Nitrotyrosine in plasma of celiac patients as detected by a new sandwich ELISA. Free Radical Biol. Med. 25:953-963.

6. Beckman, J.S., and Koppenol, W.H. 1996 Nitric oxide, superoxide, and peroxynitrite: the good, the bad, and ugly. Am. J. Physiol. 271:C1424-C1437.

7. Cuzzocrea, S., Riley, D.P., Caputi, A.P., and Salvemini, D. 2001. Antioxidant therapy: a new pharmacological approach in shock, inflammation, and ischemia/reperfusion injury. Pharmacol. Rev. 53:135-159.
8. Marfella, R., et al. 2000. Circulating adhesion molecules in humans: role of hyperglycemia and hyperinsulinemia. Circulation. 101:2247-2251.

9. Marfella, R., et al. 2000. The effect of acute hyperglycaemia on QTc duration in healthy man. Dia betologia. 43:571-575.

10. Frustaci, A., et al. 2000. Myocardial cell death in human diabetes. Circ. Res. 87:1123-1129.

Pennathur, et al. reply - The demonstration by Marfella et al. that acute hyperglycemia increases the levels of protein-bound nitrotyrosine in plasma may have important implications for the pathogenesis of vascular dysfunction in diabetics. An advantage of the ELISA used by Marfella et al. is the avoidance of acidic conditions that promote nitrotyrosine formation ex vivo $(11,12)$. The correlation between the increase in mean arterial blood pressure and nitrotyrosine levels supports the authors' proposal that nitric oxide - a potent vasodilator - is reacting with superoxide to generate reactive nitrogen species.

However, we believe that there are two important caveats regarding these interesting observations. First, as the authors acknowledge, nitrotyrosine is not a specific marker for peroxynitrite. Indeed, nitrotyrosine can be produced by peroxidases, nitrogen dioxide radical, nitrite under acidic conditions, and hypochlorous acid plus nitrite (13). Second, we believe that it is critical to confirm that nitrotyrosine is elevated using an analytical method (such as mass spectrometry) that is not potentially confounded by structurally related molecules (14).

In future studies, it will be important to establish the pathway(s) for the increase in nitrotyrosine levels during acute hyperglycemia. One potential mechanism involves glucose-stimulated mitochondrial superoxide production (3). Because vascular disease is the leading cause of death in diabetic humans, it would also be of great interest to determine whether free or protein-bound nitrotyrosine levels are increased in these individuals.

\section{Subramaniam Pennathur and Jay W. Heinecke}

1. Yi, D., Ingelse, B.A., Duncan, M.W., and Smythe, G.A. 2000. Quantification of 3-nitrotyrosine in biological tissues and fluids: generating valid results by eliminating artifactual formation. $J$. Am. Soc. Mass. Spectrom. 11:578-586.

2. Frost, M.T., Halliwell, B., and Moore, K.P. 2000 Analysis of free and protein-bound nitrotyrosine in human plasma by a gas chromatography/mass spectrometry method that avoids nitration arti facts. Biochem. J. 345:453-458

3. van der Vliet, A., Eiserich, J.P., Halliwell, B., and Cross, C.E. 1997. Formation of reactive nitrogen species during peroxidase-catalyzed oxidation of nitrite. A potential additional mechanism of nitric oxide-dependent toxicity. J. Biol. Chem. 272:7617-7625.

4. Kaur, H., Lyras, L., Jenner, P., and Halliwell, B. 1998. Artefacts in HPLC detection of 3-nitrotyrosine in human brain tissue. J. Neurochem. 70:2220-2223.

5. Nishikawa, T., et al. 2000. Normalizing mitochondrial superoxide production blocks three pathways of hyperglycaemic damage. Nature. 404:787-790. 